\title{
Internalização de novos membros em equipes de desenvolvimento de software: benefícios e limitações
}

\author{
Patrícia Cristina Moser, Joelson Isidro da Silva Araujo, Everton Renne \\ Barros de Oliveira, Henrique Santos Ferreira, Christiane Pereira
}

\author{
Centro de Informática (Cin) - Universidade Federal de Pernambuco (UFPE) - \\ Pós-graduação em Ciência da Computação \\ 50740-560 - Cidade Universitária, Recife - PE- Brasil \\ $\{p c m 3, j$ isa, erbo, hsf, cmlp, $\}$ cin.ufpe.br
}

\begin{abstract}
Context: The internalization of new members into software development teams causes changes in organizations. Objective: To investigate the benefits and limitations of internalization in software development teams. Method: A qualitative study was carried out in a software development startup where internalization was observed recently. Results: The data collected were analyzed using qualitative coding techniques and the results were verified and validated with the participants through the verification of members. Conclusion: It was noticed that the internalization is strongly influenced by the company's culture and the reception and integration of the team.
\end{abstract}

Resumo. Contexto: A internalização de novos membros em equipes de desenvolvimento de software ocasiona mudanças nas organizações. Objetivo: Investigar os beneficios e limitações da internalização em equipes de desenvolvimento de software. Método: Foi realizado um estudo qualitativo em uma startup de desenvolvimento de software onde a internalização foi observada recentemente. Resultados: Os dados coletados foram analisados utilizando técnicas de codificação qualitativa e os resultados foram verificados $e$ validados com os participantes por meio da verificação de membros. Conclusão: Percebeu-se que a internalização sofre forte influência da cultura da empresa e do acolhimento e integração do time.

Keywords: Internalização; cultura organizacional; integração; acolhimento; engenharia de software; times de desenvolvimento.

\section{Introdução}

Durante várias décadas, a pesquisa sobre gestão de recursos humanos investigou práticas para melhorar o desempenho de pessoas no trabalho. Esses estudos se concentram em indivíduos - em vez das equipes ou da organização como um todo - e consideram a experiência dos trabalhadores e as práticas de trabalho individuais como um dos principais elementos para aumentar o desempenho do trabalho (Santos et. al, 2016).

Um problema bastante comum nas equipes de desenvolvimento de software é a rotatividade de membros, seja por conta do desligamento de um membro ou por substituição/rodízio entre as equipes. Atualmente não existem estudos que avaliem quais os benefícios e limitações desta rotação entre membros nas equipes. Já é possível encontrar, de maneira ainda incipiente, trabalhos que buscaram entender como a prática de rotação influencia a motivação e satisfação dos engenheiros de software (Santos, 2015) 
ou, quais os efeitos positivos e negativos para a empresa e o engenheiro com esta rotação (Santos et. al, 2016). Desta forma é possível identificar uma oportunidade de pesquisa que busque compreender os efeitos da substituição ou inclusão de novos membros nas equipes de software, e entender como se dá a internalização deste novo membro.

Isto posto, o objetivo deste trabalho é investigar quais são os benefícios e limitações do processo de internalização de um novo membro em uma equipe de desenvolvimento de software.

Realizamos um estudo qualitativo, utilizando técnicas de codificação, com o objetivo de coletar dados sobre o processo de internalização de novos membros na startup estudada. Os dados coletados foram analisados e os resultados foram verificados e validados com os participantes através da verificação de membros. Este estudo forneceu evidências empíricas sobre os benefícios e limitações sentidas pelos integrantes da equipe, assim como pelos novos membros.

O artigo está organizado da seguinte forma: na seção 2, apresentamos o referencial teórico; na Seção 3, descrevemos a metodologia utilizada; na Seção 4, apresentamos os resultados obtidos e, finalmente, as conclusões são apresentadas na Seção 5.

\section{Referencial Teórico}

Nesta seção serão apresentados os aspectos que embasam este estudo.

\subsection{Fatores Humanos em Engenharia de Software}

A literatura de gestão aponta que pessoas são o principal contribuinte para o sucesso de projetos (Tyler, 1997). O desenvolvimento de software é uma atividade intelectual que depende essencialmente de pessoas, que geralmente formam equipes e trabalham juntas para entregar os softwares ou serviços contratados (Miranda, 2011).

É impossível excluir os fatores humanos durante o desenvolvimento de software, porque o software é desenvolvido por pessoas e para elas. A natureza intangível do software tornou um produto difícil de ser criado com sucesso, e um exame minucioso das razões para as principais falhas no sistema de software mostra que várias dessas razões estão relacionadas a problemas humanos (Capretz, 2017). Mas os fatores humanos, como capacidade do time e experiência, também são apontados como principais responsáveis por atrasos nos projetos (Tyler, 1997).

\subsection{Cultura Organizacional}

Cultura Organizacional é um padrão de pressupostos que um determinado grupo criou, descobriu ou desenvolveu durante seu processo de aprendizagem para lidar com seus problemas de adaptação externa e integração interna, e que funcionou bem o suficiente para ser considerado válido e, portanto, para ser ensinado a novos membros como a maneira correta de perceber, pensar e sentir em relação a esses problemas (Schein, 1984).

Segundo Berger e Luckman (1984), a cultura é socialmente construída e é o produto de escolhas explícitas e implícitas que são impostas ou renegociadas e expressas através da linguagem e da ação. Além disso, a cultura tem sido vista como um elemento influenciador que afeta a moral do empregado, sua motivação e disposição; nível de produtividade e eficácia; a qualidade do trabalho; inovação e criatividade e a atitude dos 
funcionários no local de trabalho (Campbell et. al, 1999). Ainda, Cameron e Quinn (2006) argumentam que a cultura é uma chave para a vantagem competitiva.

\subsection{Integração e Acolhimento}

Nos modelos de gestão atuais um dos maiores focos de preocupação é com a gestão do capital humano, ou seja, a sensação de bem-estar e motivação dos colaboradores. Esta mudança de paradigma é explicada pelo fato de o capital humano ser considerado como o motor principal para o sucesso organizacional, ainda que não seja fácil quantificar qual o valor dessa contribuição para a concretização dos resultados (Cunha, 2014).

A formação de acolhimento e integração de novos trabalhadores pode ser um dos primeiros passos para criar laços de identidade entre o trabalhador e a organização. Eles mais facilmente se adequam à cultura organizacional, aos padrões de comportamento considerados aceitáveis pela mesma, aos colegas de trabalho e à liderança.

Atualmente vem se valorizando cada vez mais a necessidade de criação de um clima organizacional onde as pessoas possam se sentir valorizadas. Há premissas que pessoas motivadas produzem mais. Isso vem se tornando tão importante que práticas motivacionais se tornaram indispensáveis para o diferencial competitivo.

Por outro lado, a integração de um novo colaborador por si só já constitui uma mudança na organização, podendo conduzir a alguma instabilidade e à atitude de desconfiança por parte de colegas, como o receio de que o novo colaborador possa interferir nas expectativas dos colaboradores já existentes. Porém é o processo de interação entre os diversos elementos, especialmente o posicionamento do novo colaborador face aos colegas, que direciona a forma como a relação irá se estabelecer. Em alguns ambientes mais hostis, atitudes de antipatia e segregação do novo colaborador podem contribuir para que se sinta discriminado e não consiga se integrar ao grupo.

\subsection{Internalização}

O termo internalização tem diferentes significados dependendo da área em que é utilizado. Na sociologia e outras ciências sociais, vertentes mais próximas da prática estudada, internalização significa a aceitação de um indivíduo para um conjunto de normas e valores estabelecidos por outros através da socialização.

John F. Scott (1971) descreveu a internalização através de uma metáfora, na qual algo (ex. uma ideia, conceito, ação) se move de fora da mente ou personalidade para um local dentro dela. Este processo de internalização começa com o aprendizado sobre as normas, em seguida, o indivíduo passa pelo processo de entender por que elas são importantes e por que fazem sentido, até finalmente aceitar as normas com seu próprio ponto de vista. O processo de internalização também leva a sentimentos de obrigação moral em respeito ao bem estar do grupo e vão além da simples troca de favores, levando a ações proativas em prol do grupo e seus membros (Tyler, 1997).

\subsection{Trabalhos Relacionados}

A literatura apresenta várias definições para descrever a prática da internalização em equipes de desenvolvimento de software. Algumas destas são encontradas nos trabalhos relacionados desta pesquisa ao abordarem job rotation, um modelo denominado developer joining model e a internalização em equipes globais. Entretanto, neste estudo investigamos o benefícios e limitações do processo de internalização em startups, uma 
vez que, é crucial o entendimento deste processo pelas empresas de software, tanto para estabelecer funcionários novos como para aproveitar ao máximo funcionários experientes.

No trabalho de Santos et. al (2016), os autores investigaram os efeitos do job rotation em uma organização de software que utiliza esta prática. Os resultados mostram que a rotação de empregos é uma importante prática organizacional que produz resultados positivos. No entanto, os gerentes devem estar cientes dos possíveis efeitos negativos e implantar táticas para equilibrá-los.

Em Steinmacher et. al (2014) foi apresentado um modelo, denominado developer joining model, que visa representar os estágios comuns e as forças que influenciam os recém-chegados a serem atraídos ou afastados de um projeto de código aberto. É útil para que as comunidades de Open source software (OSS) possam planejar investimentos para melhor apoiar a adesão de novos membros.

Já Fagerholm e Guinea (2014) investigaram os impactos da internalização de membros em equipes de software globais. Para os autores, o problema geral de introduzir novas pessoas em uma organização existente é especialmente pronunciado em ambientes distribuídos com equipes virtuais. Os autores afirmam que o suporte dado aos desenvolvedores, como por exemplo a mentoria, influencia positivamente no processo de integração.

\section{Método}

Nesta pesquisa, estamos interessados em entender o processo de internalização de novos membros em equipes de desenvolvimento de software e quais os benefícios e limitações desse fenômeno. Foi realizada uma pesquisa qualitativa que, de acordo com Merriam (2009), ao invés de determinar a causa e efeito de alguma variável, ou de prever ou descrever a distribuição de algum atributo entre uma população, este tipo de pesquisa está interessada em descobrir o significado de um fenômeno para os envolvidos, e entender como as pessoas interpretam suas experiências e o significado que atribuem à elas.

\subsection{Questão de Pesquisa}

Conforme Gil (2010), toda pesquisa se inicia com algum tipo de problema, ou indagação e o mesmo deve ser formulado como pergunta. Para compreender o fenômeno estudado, foi formulada a seguinte questão de pesquisa, a qual este estudo visa responder:

QP: Quais os benefícios e limitações da internalização de membros em equipes de desenvolvimento de software?

A resposta para esta questão de pesquisa é apresentada na Seção 4.

\subsection{As fases da pesquisa}

Inicialmente foi realizada uma busca, na literatura, por estudos que pudessem embasar o nosso referencial teórico. A busca foi realizada de maneira adhoc.

Em paralelo com o estudo inicial, foi elaborado um roteiro de entrevistas para a coleta de dados. Após um pré-teste realizado, foi possível identificar e eliminar possíveis problemas. A realização do pré-teste resultou em alterações que proporcionaram o aprimoramento do instrumento em relação à ordem das questões e ao vocabulário 
utilizado, facilitando a aplicação do roteiro. Com o roteiro ajustado fomos a campo para a coleta dos dados. Por fim, procedeu-se a análise dos resultados e redação deste trabalho.

\subsection{Descrição da Empresa e dos participantes}

A empresa selecionada é uma startup pernambucana de tecnologia, fundada em 2012, especializada em desenvolvimento de softwares com foco no mercado de comunicação e marketing. Atualmente, a startup possui 24 funcionários. Destes, 7 atuam na área de desenvolvimento.

Pela própria característica da startup, que mantém um crescimento contínuo da equipe de desenvolvimento, o fenômeno da internalização é observado com frequência. O tempo de empresa entre os entrevistados varia de 01 mês à 18 meses.

Da formação inicial da equipe, apenas o Product Owner (PO) ainda está do time. Todos os outros participantes foram substituídos. Na entrevista com o PO foi observada uma forte questão cultural no processo seletivo, onde os valores de colaboração e de espírito de equipe são fundamentais para que o participante permaneça na equipe.

\subsection{Elaboração do Instrumento}

De acordo com Merriam (2009), recomenda-se o uso de múltiplos métodos para a coleta de dados, entre eles as entrevistas, análise de documentos, questionários e observações. Porém, na ausência dos outros métodos, todos os dados são coletados através de entrevistas, o método que utilizamos neste estudo.

Foram realizadas entrevistas semi-estruturadas com um grupo de desenvolvedores de software. De acordo com Merriam (2009), a maior parte de uma entrevista semiestruturada é guiada por uma lista de perguntas ou questões a serem exploradas. Este formato permite ao pesquisador responder à situação em questão, à visão de mundo emergente do entrevistado e a novas ideias sobre o tema.

O roteiro de entrevistas seguiu as recomendações propostas por Merriam (2009), onde as perguntas estão no centro das entrevistas e para coletar dados significativos, o pesquisador deve fazer boas perguntas. As questões mais abertas são as melhores de serem utilizadas. Além disso, ter menos perguntas, porém mais abertas, faz com que o pesquisador possa ser flexível no uso do roteiro, permitindo que o pesquisador ouça o que seu entrevistado tem para compartilhar. Um pré-teste da entrevista foi realizado, buscando validar o roteiro. Dessa forma, foi possível ajustar o script, proporcionando um aprimoramento do instrumento. Com o pré-teste também foi possível estimar o tempo das entrevistas, que ficou em torno de 25 minutos.

\subsection{Iniciando a pesquisa de campo}

Inicialmente foi escolhida a empresa para a pesquisa de campo. Essa escolha foi motivada por dois fatores: 1. o fenômeno da internalização ter ocorrido recentemente e 2 . por termos acesso total a todos os dados e indivíduos necessários para nossa investigação.

\subsubsection{Seleção dos Participantes}

Como optamos pela escolha de uma startup em tecnologia, todos os colaboradores que fazem parte da equipe de desenvolvimento foram entrevistados. Isso inclui o gerente de projeto e os demais desenvolvedores. Como o ambiente de pesquisa foi uma startup em 
crescente desenvolvimento, a prática da internalização foi verificada em vários momentos desde a formação inicial da equipe.

\subsubsection{Entrevistas}

Todas as 07 entrevistas foram realizadas por um entrevistador e apoiadas por um segundo entrevistador, que fazia notas de campo como forma de auxiliar na análise dos dados. Todas os entrevistados autorizaram a gravação das entrevistas, as quais duraram, em média, cerca de 20 minutos, com exceção da entrevista com o gerente de projetos, que levou 36 minutos. Foram produzidos 150 minutos de áudio e 62 páginas de transcrições. Todas as entrevistas ocorreram nas instalações da startup onde o fenômeno foi observado.

\subsection{Análise dos dados}

O objetivo da análise qualitativa é consolidar, reduzir e interpretar dados obtidos de várias fontes, e dar sentido a eles (Merriam, 2009). Envolve rotular e codificar todos os dados, a fim de identificar semelhanças e diferenças para descrever o fenômeno em estudo. Em nosso estudo, a análise de dados foi realizada em paralelo com a coleta de dados, em etapas incrementais e iterativas, seguindo as recomendações de Merriam (2009).

Dessa forma, nossa análise de dados começou com a codificação aberta das transcrições. Com a leitura da primeira transcrição, os códigos iniciais foram definidos. A cada leitura de novas transcrições, os códigos decorrentes de cada entrevista foram constantemente comparados aos códigos das outras entrevistas. A partir das constantes comparações dos códigos, as categorias foram formadas. À medida que o processo de análise de dados progrediu, as relações entre categorias foram construídas.

Por fim, as categorias principais foram escolhidas de acordo com sua relevância dentro do contexto do fenômeno da internalização. A partir da definição das categorias centrais, foram definidas as proposições, o que possibilitou a interpretação das relações no fenômeno estudado, apresentada na seção 4.

Neste ponto, consolidamos os resultados e usamos técnicas de verificação de membros para validar esses achados, melhorando a precisão, credibilidade e validade interna de nossas interpretações (Harper and Cole, 2012; Kreftin, 1991). Os resultados foram apresentados a 4 dos 7 respondentes e nenhum deles discordou dos achados.

\section{Resultados}

Nesta seção detalharemos as categorias centrais do nosso trabalho que foram obtidas das entrevistas com os 7 participantes, junto com a construção das relações entre essas categorias. Nós usamos a técnica de codificar, categorizar e sintetizar os dados com o intuito de construir a narrativa central que explica os benefícios e limitações do processo de internalização de um novo membro em uma equipe de desenvolvimento de software.

O primeiro passo foi realizar as entrevistas e transcrevê-las. A partir das transcrições separamos os códigos de cada uma das entrevistas, e posteriormente, elencamos os códigos únicos e comuns a cada uma delas. Depois partimos para verificar os códigos que possuem semântica similar e os agrupamos, para dar início a criação das categorias. Por fim definimos a relação que existe entre essas categorias para, a partir desse desenho, criarmos a narrativa que responde nossa pergunta de pesquisa.

No nosso estudo, encontramos duas categorias centrais: 1. Cultura da empresa (pelo lado da empresa) e 2. Acolhimento / integração do time (pelo lado da equipe). 
Abaixo seguem as relações que encontramos entre essas duas categorias e também outras relações entre outras categorias, que foram identificadas no nosso trabalho.

Relação 1 - Constatamos que a cultura da empresa influencia diretamente o acolhimento e integração do time.

"A entrada de um novo membro só tende a.. como é que eu posso dizer? Influenciar nessa cultura, afinal a cultura é feita pelo próprios (membros). É o próprio time".

"Todo mundo praticamente é uma família, tá todo mundo junto ali, disponivel para ajudar um ao outro e tirar dúvidas, enfim. Eu acho que isso é o grande diferencial de trabalhar aqui".

O membro internalizado tem que se adequar a essa cultura para ser aceito. Se ele tem as características necessárias para essa forma de integração, ele permanece no grupo e é acolhido pelo mesmo. Caso contrário, apesar de o sentimento de perda dos demais, por todos "serem uma família", o membro termina sendo expurgado naturalmente, e pelo fato de ele não estar agregando valor, essa saída termina sendo aceita pelos que ficam.

Relação 2 - A cultura também influencia os treinamentos.

"Eu não tô conseguindo treinar ele da forma como deveria por conta de tempo mesmo, e.. acaba que o resto do time percebeu isso e tá ajudando muito ele".

A cultura da empresa faz com que a pessoa que entra tenha tempo dedicado ao aprendizado sem cobranças iniciais por produtividade. O processo de produção só tem início após um período de treinamento inicial para adaptação e acompanhamento.

Relação 3 - Os treinamentos influenciam a expectativa do trabalho.

"Será que os sócios tão sabendo que eu tô em treinamento ainda?"

"Tô me sentindo meio improdutivo, porque eu acho que o pessoal tá esperando mais de mim."

Há uma preocupação e um sentimento de insegurança de quem chega para saber se está atendendo às expectativas. $\mathrm{O}$ membro internalizado acha que não está dando conta das demandas. Ele não leva em consideração que ainda está em fase de treinamento e que a cultura da empresa permite esse tempo inicial "improdutivo".

Relação 4 - os treinamentos influenciam o acolhimento/integração.

"Confortável. Eh, bem acolhido pelas pessoas e confortável para trabalhar também, tecnicamente falando".

A medida que as pessoas conhecem e estão capacitadas para realizar todo o processo técnico do trabalho isso facilita bastante o processo de integração, pois eles se sentem mais confiantes e confortáveis junto a todo o grupo. Elas passam a falar a mesma língua e possuem um sentimento de fazer parte do grupo de forma mais rápida.

Relação 5 - O acolhimento/integração influencia na divisão da carga de trabalho.

"Eu acho que dentro da equipe ele meio que desafogou o outro "Back-end", pois era muito atarefado e a gente tava num momento de transição de infraestrutura e ai ele meio que começou... eu senti que a chegada dele começou a diminuir esses gargalos".

"Com a minha chegada é exatamente isso que a gente quer, conseguir estabilizar esse problema com menos tempo e acredito que esteja sendo favorável".

A internalização do novo membro foi vista por todos como uma possibilidade de melhor divisão da carga de trabalho e uma expectativa de que a carga de cada um poderia diminuir. Nas nossas entrevistas levantamos que as pessoas se sentiam motivadas, e até 
emocionadas com o acolhimento de todo o grupo durante a internalização, e isso gerava uma melhor adaptação, que pode ajudar a diminuir os gargalos, facilitando a redução da carga de trabalho para todos. saída.

Relação 6 - O acolhimento/integração influencia sentimento de perda devido a

"Não é um sentimento agradável, lógico. São membros que a gente trabalhava e tinha uma relação de amizade, mas é um sentimento que olhando pelo lado de equipe, é um sentimento bom... Então quando o pessoal que não cumpre esse papel é desligado, é.. e a gente consegue suprir essas necessidades com pessoas que cumpram o papel com excelência, acaba que o peso que ficava pra você, é dividido em igual com todos os membros".

Há um impacto com a saída dos membros, no entanto, como a visão de grupo é mais forte, quando se percebe que a pessoa não está realizando seu papel e isso sobrecarrega o resto do grupo, a saída desse membro passa a ser vista como uma melhoria e o impacto é suavizado e dividido com todos os membros. O sentimento passa a ser positivo, pois fica claro que o membro está atrapalhando todo o time. Isso mostra claramente que há uma seleção natural para manter o grupo junto no mesmo objetivo.

Relação 7 - A cultura influencia o processo de desenvolvimento.

$\mathrm{Na}$ empresa foi escolhida uma metodologia ágil de desenvolvimento e foi definido o modelo rotativo scrum master, para que todos possam experimentar papéis diferentes, como também a programação em pares para que o acolhimento e a capacitação fiquem ainda mais fortes.

Relação 8 - O processo de agregar valor com contribuição influencia o processo de desenvolvimento.

O processo de agregar valor, melhorando qualidade das entregas, influencia o processo de desenvolvimento, pois reforça a necessidade de um método que torne todo o processo mais seguro e ágil para que as expectativas sejam atendidas. Para que isso ocorra é importante a definição clara dos papéis e responsabilidades, que ficam bem definidas no processo de desenvolvimento escolhido. Todos os entrevistados, ao descrever a equipe, tinham a visão clara de qual seriam as contribuições do novo membro para a equipe, além de ter conhecimento sobre o papel e responsabilidade de cada membro.

Relação 9 - A cultura influencia a abertura do diálogo.

A cultura, baseada no processo de organização horizontalizada e com uma visão mais informal, influencia a abertura do diálogo do grupo, o processo de desenvolvimento escolhido, os treinamentos iniciais (para prover mais capacitação aos membros novos) e o acolhimento/integração (que facilita o processo de internalização).

Dessa forma podemos perceber que a cultura da empresa é um fator determinante na internalização de novos membros. Quando a pessoa que está entrando se adequa à cultura, ocorre uma melhoria nas entregas, com muito mais valor agregado, sendo o processo de acolhimento muito positivo para todos. Por outro lado, percebe-se uma limitação: a de que os novos membros precisam se enquadrar nesse modelo, ou não serão aceitos. Quando um novo membro entra para o time, existe um forte acolhimento, mas se a pessoa não está contribuindo com a equipe, ela é substituída. A visão de grupo está sempre em primeiro lugar. 


\section{Conclusão}

Nossos resultados mostram os benefícios e limitações da internalização em membros em equipes de desenvolvimento de software. A empresa estudada foi uma startup de tecnologia que desenvolve softwares com foco no mercado de comunicação e marketing. Utilizamos técnicas de pesquisa qualitativa para coletar, analisar e sintetizar dados através de entrevistas para a realização do nosso trabalho.

Nossos resultados mostraram que a cultura da empresa influencia diretamente no acolhimento dos novos membros, e consequentemente, na integração de todo o grupo. Mostrou também que o novo membro, para ser aceito, tem que se adequar a essa cultura. No nosso caso, o processo de internalização buscou a formação de um novo grupo com características mais homogêneas e mais acolhedoras.

Essa cultura mais acolhedora permite que os novos membros possam ter um tempo inicial dedicado a aprendizagem, e define claramente o papel e responsabilidade de cada um, para garantir um melhor resultado de todos os trabalhos. Todo esse processo faz com que as pessoas se sintam mais confiantes e confortáveis com os seus desafios individuais, e isso tende a levar ao aumento da produtividade de todo o grupo ao longo do tempo. Outro ponto importante é que essa cultura de acolhimento também ajuda na divisão da carga de trabalho, o que ajuda a diminuir os gargalos existentes e a balancear melhor a carga de trabalho. Toda essa estrutura ajuda na motivação geral do grupo e no fortalecimento da visão da equipe.

Reforçamos, mais uma vez, que a cultura da empresa acolhedora exerce forte influência e facilita a internalização de novos membros. Com a entrada de um membro que esteja alinhado à cultura de colaboração, é percebido que ocorre melhoria nas entregas com mais valor agregado, diminuindo gargalos e aumentando a produtividade e motivação de todos na busca de melhores resultados.

Por outro lado, o processo se torna mais fechado, uma vez que exige um perfil específico do novo membro. Se esse membro não se adapta, e se percebe que ele não está contribuindo como se esperava, ele naturalmente é expelido do grupo, e apesar do sentimento de perda que isso gera, esse sentimento é compensado pela expectativa de ganho que o grupo vai ter já que a contribuição dada pelo membro está fora do esperado.

Com base na nossa observação, entendemos que a internalização de novos membros, se feita de forma a garantir uma maior homogeneidade do grupo, gera uma maior sinergia, que ajuda fortemente no objetivo dos resultados organizacionais. É necessário ficar atento nesse processo para não criar um grupo tão fechado que dificulte a aceitação de novos membros, já que o processo de mudança sempre vai ocorrer.

Por fim, é importante ressaltar que todos os achados se referem à empresa estudada, o que nos impossibilita de fazer generalizações. Outros estudos, com amostras maiores e com um número maior de observações, são necessários.

\section{AGRADECIMENTOS}

Agradecemos à CAPES e à FACEPE pelo apoio financeiro ao desenvolvimento de pesquisas de pós-graduação, cedido por meio de bolsas de estudo, para Joelson Isidro da Silva Araujo (CAPES), Everton Renne Barros de Oliveira (CAPES) e Henrique Santos Ferreira (FACEPE). 


\section{Referências}

Berger, P. e Luckman, T. (1984). The social construction of reality: a treatise in the sociology of knowledge. London: Penguin Books.

Cameron, K.S. Quinn, R.E. (2006). Diagnosing and Changing Organizational Culture, Jossey-Bass, San Francisco, CA.

Campbell, D., Stonehouse, G., Houston, B. (1999). Business Strategy an Introduction, Butterworth- Heinemann, Oxford.

Capretz, L.F., Ahmed, F., Silva da, F.Q.B. (2017). Soft Sides of Software. Information and Software Technology.

Cunha, A. (2014). A arte da guerra na liderança (2 Ed). Lisboa: TopBooks

Fagerholm, F. and Guinea, A. S. (2014). Onboarding in Open Source Projects. IEEE Software(Volume: 31 , Issue: 6 , Nov.-Dec. 2014 ).

Gil, A. C. (2010). Como elaborar projetos de pesquisa. 5. ed. - São Paulo: Atlas.

Harper, M.; Cole, P. Member Checking: Can Benefits Be Gained Similar to Group Therapy? The Qualitative Report, 2012.

Krefting, L. Rigor in qualitative research: The assessment of trustworthiness. The American Journal of Occupational Therapy, 1991.

Merriam, S. B. (2009). Qualitative research: A guide to design and implementation. Journal of Chemical Information and Modeling.

Miranda, R. (2011). Uma Revisão Sistemática Sobre Equipes de Desenvolvimento de Software: Tipologia, Características e Critérios de Formação. Dissertação de mestrado, Universidade Federal de Pernambuco.

Santos, R. E. S., Silva, F. Q. B., Magalhães, C. V. C. and Monteiro, C. V. F. (2016). Building a theory of job rotation in software engineering from an instrumental case study. IEEE/ACM 38th IEEE International Conference on Software Engineering.

Santos, R. E. S. (2015). The Influence of Job Rotation on Motivation and Satisfaction of Software Engineers. Master Thesis in Computer Science. Federal University of Pernambuco, Brazil.

Steinmacher, I., Gerosa, M. A., Redmiles, D. (2014). Attracting, Onboarding, and Retaining Newcomer Developers in Open Source Software Projects. Workshop on Global Software Development in a CSCW Perspective, held in Conjunction with the 17th ACM Conference on Computer Supported Cooperative Work \& Social Computing(CSCW'14), 2014.

Schein, E. (1984), Coming to a new awareness of Organizational Culture, Sloan Management Review, Vol. 25, No. 2, 3-16.

Scott, J. (1971). Internalization of Norms: A sociological Theory of Moral Commitment.

Tyler, T. R. (1997). Why people cooperate with organizations. Research in Organization Behavior, 21, 201-246.

Whitehead, J. (2007). Collaboration in Software Engineering: A Roadmap. Future of Software Engineering. IEEE - FOSE'07. 